\title{
Cationic Au Nanoparticle Binding with Plasma Membrane-like Lipid Bilayers: Potential Mechanism for Spontaneous Permeation to Cells Revealed by Atomistic Simulations
}

\author{
Elena Heikkilä, ${ }^{\dagger, \dagger}, \nabla$ Hector Martinez-Seara, ${ }^{\dagger, \nabla}$ Andrey A. Gurtovenko, ${ }^{\S} \|$ Matti Javanainen, ${ }^{\dagger}$ \\ Hannu Häkkinen, ${ }^{\#}$ Ilpo Vattulainen, ${ }^{\dagger, \&}$ and Jaakko Akola*,†,‡,@ \\ ${ }^{\dagger}$ Department of Physics, Tampere University of Technology, P.O. Box 692, FI-33101 Tampere, Finland \\ ${ }^{\ddagger}$ COMP Centre of Excellence, Department of Applied Physics, Aalto University, FI-00076 Aalto, Finland \\ ${ }^{\S}$ Institute of Macromolecular Compounds, Russian Academy of Sciences, Bolshoi Prospect 31, V.O., St. Petersburg 199004, Russia \\ "Department of Molecular Biophysics, Faculty of Physics, St. Petersburg State University, St. Petersburg 198504, Russia \\ \# Departments of Physics and Chemistry, Nanoscience Center, University of Jyväskylä, FI-40014 Jyväskylä, Finland \\ ${ }^{\&}$ Center for Biomembrane Physics (MEMPHYS), University of Southern Denmark, Odense DK-5230 Denmark \\ ${ }^{\circledR}$ PGI-1, Forschungszentrum Jülich, D-52425 Jülich, Germany
}

Supporting Information

ABSTRACT: Despite being chemically inert as a bulk material, nanoscale gold can pose harmful side effects to living organisms. In particular, cationic $\mathrm{Au}$ nanoparticles $\left(\mathrm{AuNP}^{+}\right)$of $2 \mathrm{~nm}$ diameter or less permeate readily through plasma membranes and induce cell death. We report atomistic simulations of cationic Au nanoparticles interacting with realistic membranes and explicit solvent using a model system that comprises two cellular compartments, extracellular and cytosolic, divided by two asymmetric lipid bilayers. The membrane-AuNP ${ }^{+}$binding and membrane reorganization processes are discovered to be governed by co-

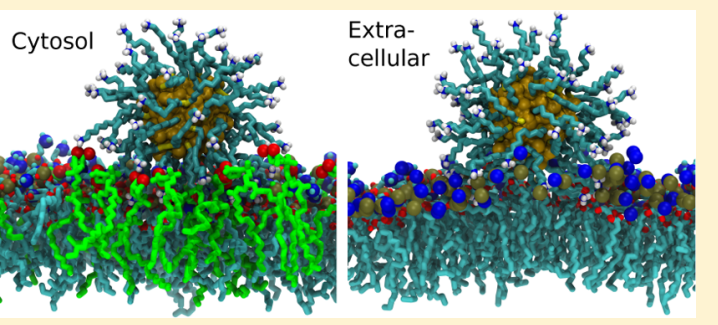
operative effects where AuNP ${ }^{+}$, counterions, water, and the two membrane leaflets all contribute. On the extracellular side, we find that the nanoparticle has to cross a free energy barrier of about $5 k_{\mathrm{B}} T$ prior forming a stable contact with the membrane. This results in a rearrangement of the zwitterionic lipids and nanoparticle side groups in the contact area, giving rise to the initial stage of pore formation on the membrane surface. Such behavior is not seen on the cytosolic side, where AuNP ${ }^{+}$is spontaneously captured by the negatively charged phosphatidylserine lipids that diffuse to enrich the membrane leaflet underneath AuNP ${ }^{+}$, further pointing to $\mathrm{AuNP}^{+}$accumulation on the inner leaflet of a plasma membrane. The results suggest AuNP ${ }^{+}$permeation to take place through the formation of a pore together with partial nanoparticle neutralization/deprotonation, leading to membrane disruption at higher nanoparticle concentrations. The data also suggest a potential mechanism for cytotoxicity as AuNP ${ }^{+}$binding to the extracellular leaflet may trigger apoptosis through translocation of phosphatidylserine.

\section{INTRODUCTION}

Metal nanoparticles (NPs) provide new functionalities of matter at the nanoscale. Their properties can be tuned via chemical composition, size, binding strength between the core and ligand shell, overall charge, and stability in a given medium. ${ }^{1}$ Quantum confinement effects arise as the NP size decreases down to a few nanometers, which is reflected, e.g., in optical properties and catalytic reactivity. ${ }^{2-4}$ In this context, gold nanoparticles (AuNPs) are among the most studied systems as they have potential for applications in molecular electronics, molecular recognition, catalysis, biolabeling and sensing, and drug delivery. ${ }^{1,3-7}$

Medical applications of AuNPs cover practically all fields, including diagnostics, therapy, prophylaxis, and hygiene. ${ }^{8}$ AuNPs can be used as intrinsic drug agents or drug delivery vehicles, and they can be applied as stabilizing agents for other drug delivery vehicles such as vesicles. AuNPs have demonstrated applicability in photothermal therapy due to their optical properties which enable local heating at cellular level. As composite materials AuNPs may function as triggers for drug release. For example, they enable an efficient treatment of diseased sites (tumors) with small side effects in the body as the local AuNP concentration is high only at the targeted site. 9 This approach is based on size-selective accumulation of AuNPs due to the disordered vascular characteristics of tumors, and the targeting effects can be further enhanced by covering nanoparticles by additional recognition units which are selective for specific tumor sites. ${ }^{9}$

Nanotoxicology is a special field of toxicology which considers potential harmful effects of NPs in living organisms. ${ }^{10}$

Received: March 10, 2014

Revised: April 30, 2014

Published: April 30, 2014 
These effects differ from those of larger particles as NPs are able to translocate inside the body from the site of deposition (e.g., lungs) to different organs and tissues (such as bloodbrain barrier) more effectively. The important factors for NP toxicity are particle size, composition, shape, surface modification, concentration, agglomeration, and solubility. ${ }^{11}$ For example, the large surface-to-volume ratio of NPs is related to increasing chemical reactivities, leading to enhanced formation of reactive oxygen species which may cause damage to proteins, DNA, and cell membranes via oxidative stress. ${ }^{10}$ Recently, AuNPs have also been found to enhance the formation of amyloid fibrils that is known as a fundamental step in Alzheimer's and Parkinson's diseases. ${ }^{12,13}$

Small cationic Au nanoparticles of diameters less than $2 \mathrm{~nm}$ are able to penetrate cell membranes, and they can be extremely toxic. ${ }^{4,6,14-16}$ This has been demonstrated, e.g., for $1.4 \mathrm{~nm}$ AuNPs which were observed to cause necrosis and mitochodrial damage to various cell lines. ${ }^{17}$ Experimental results indicate that the nanoparticle translocation occurs via self-penetration, where the charge and specific structure/ composition of the ligand shell affect the process: Cationic AuNPs with an alternating pattern of aliphatic (hydrophobic) and functionalized side groups (striped AuNPs) show increased penetration activity in comparison to randomly distributed side groups. ${ }^{18}$ Cationic nanoparticles have been reported to generate holes in both model and living cell membranes, where the phase of the lipid bilayer plays a role for the level of disruption. ${ }^{14,19-21}$ Most recently, it was shown that striped anionic AuNPs (with amphiphilic surface) can pass nondisruptively through model membranes, and this activity depends closely on the AuNP diameter. ${ }^{22}$

Obviously, the spontaneous permeation of AuNPs and its effect on cell membranes call for urgent attention to assess the potential risks of AuNPs for future biomedical applications. As cells and cellular compartments are surrounded by membranes, it is highly relevant to investigate the interaction of AuNPs with lipid bilayer surfaces at the atomistic level. Our computer simulations focus on this issue in detail as we test the selfpenetration theory and shed light on the source of the cytotoxicity of AuNPs.

Recent experiments by Tatur et al. for model membrane systems provide a valuable reference for simulations, ${ }^{23}$ as they considered $2 \mathrm{~nm}$ diameter AuNPs floating between singlecomponent bilayers composed of zwitterionic DSPC lipids (1,2-distearoyl-sn-glycero-3-phosphocholine). On the basis of neutron reflectometry measurements, they concluded that AuNPs with cationic head groups penetrate inside the hydrophobic interior of the lipid bilayers and disrupt the membranes at increased concentrations, while anionic AuNPs stay outside. The penetration of cationic AuNPs occurs after elevating the temperature up to $53{ }^{\circ} \mathrm{C}$, which suggests that the process requires the crossing of a free energy barrier at physiological temperatures. These findings can be compared straightforwardly with simulations as there are no complications arising from other components that exist in real membranes, such as membrane proteins and the protruding glycocalyx network.

The primary objective of our work is to unlock the atomistic details of complexation between AuNPs and plasma membrane -like lipid membranes, determine how the complexation can possibly alter cell function, and evaluate how AuNPs can permeate spontaneously through plasma membranes. For this purpose, we have performed a series of atomistic molecular dynamics (MD) simulations of a monolayer-protected $\mathrm{AuNP}^{+}$ $\left[\mathrm{Au}_{144}(\mathrm{SR})_{60}\right.$, where $\left.\mathrm{R}=-\left(\mathrm{CH}_{2}\right)_{11}-\mathrm{NH}_{3}{ }^{+}\right]{ }^{24-27}$ The simulations have been carried out in an aqueous solution in the presence of a model lipid bilayer mimicking the plasma membrane, where we differentiate between the extracellular (EC) and intracellular/cytosolic (IC) leaflets. To our knowledge, this is the first attempt to tackle this complex problem at the atomistic scale, and we pay particular attention to the role of counterions, water, and specific lipid molecules to gain a full understanding of electrostatics that is expected to be important in the binding and permeation events. Importantly, our work provides a great deal of added value to previous theoretical work on AuNPs interacting with lipid bilayers, as previous studies ${ }^{28-33}$ have been based on coarse-grained (CG) models that lack the atomistic details for AuNP as well as the hydrodynamic degrees of freedom. Meanwhile, earlier atomistic studies of AuNPs have explored only the role of solvent, ${ }^{34,35}$ including our recent work that is the basis of this study. ${ }^{36}$

\section{- COMPUTATIONAL MODELS AND METHODS}

Cells use compartmentalization to create specific environments to perform their vital functions, and they achieve this objective by using membranes. In our context, the most important one is the plasma membrane, which separates cells from their surroundings. Cationic gold nanoparticles $\left(\mathrm{AuNP}^{+}\right)$are known to be able to reach the interior of cells, ${ }^{11,12,14,19,37,38}$ yet the mechanism how they do it is not known.

For studying the AuNP ${ }^{+}$-plasma membrane interaction, we have designed two model systems described in Figure 1. Essentially, our models are double bilayer systems with periodic boundary conditions applied in all directions (Figure $1 \mathrm{~b}$ ). This setup effectively provides us with two independent compartments in each system. ${ }^{33,39}$ We mimic the extracellular and intracellular/cytosolic environments by using asymmetric bilayers with lipid compositions resembling eukaryotic plasma membranes with different ion compositions in each compartment (EC, IC). The lipid composition in the outer EC leaflet is described by zwitterionic POPC [1-palmitoyl-2-oleoyl-snglycero-3-phosphocholine], while a mixture of POPC (81.25\%) and negatively charged POPS [1-palmitoyl-2-oleoylsn-glycero-3-phosphoserine] (18.75\%) is used to model the inner IC leaflet. The upper and lower membranes are inverted, and this results in an EC compartment only in contact with the pure POPC leaflets and an IC compartment in contact with the POPC/POPS mixture. Cholesterol, one of the abundant lipid types in plasma membranes of eukaryotic cells, was not included in the model since (for computational efficiency) we wanted to speed up the dynamics that is slowed down by cholesterol.

Further, a cationic gold nanoparticle with a $2 \mathrm{~nm}$ core diameter (144 Au atoms and 60 side groups with positively charged amine terminals) with $\mathrm{Cl}^{-}$counterions is placed in either the EC or IC compartment (Figure 1c,d). We compensate for the negative charge of POPS by including additional $\mathrm{K}^{+}$ions in IC. Some simulations have been performed also with salt by placing $150 \mathrm{mM}$ of $\mathrm{NaCl}$ and $\mathrm{KCl}$ in the $\mathrm{EC}$ and IC compartment, respectively, in order to mimic the physiological ion distribution in both cases.

All MD simulations were performed with the GROMACS simulation package (versions 4.0.5, 4.6.3). ${ }^{40}$ Each asymmetric bilayer contains in total 252 lipids in the double bilayer setup (see Figure 1). The lipid compositions of each leaflet has been chosen to be representative of the outer leaflet (124 POPC) 

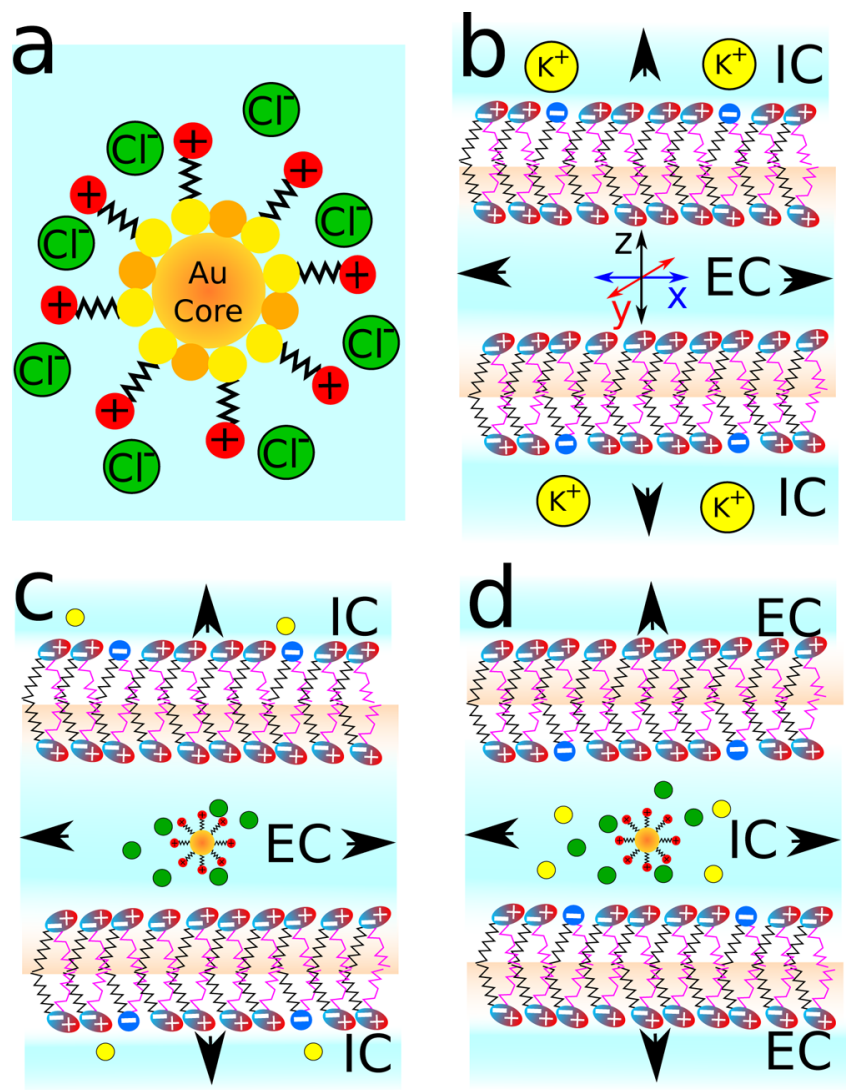

Figure 1. Schemes of the different models simulated: (a) $\mathrm{AuNP}^{+}$in aqueous solution; (b) double asymmetric bilayer; (c) $\mathrm{AuNP}^{+}$in EC compartment; and (d) $\mathrm{AuNP}^{+}$in IC compartment. Color code: $\mathrm{AuNP}^{+}$[Au (orange), S (yellow), alkyl chain (black), primary amine (red)]; lipids [chains: palmitoyl $s n-1$ chain (black), oleyol $s n-2$ chain (magenta); head groups: phosphatidylserine (blue), phosphatidylcholine (cyan/red)]; ions $\left[\mathrm{Cl}^{-}\right.$(green), $\mathrm{K}^{+}$(yellow)]. EC refers to the "extracellular" and IC to the "intracellular" compartment. The leaflet next to the EC regime corresponds to pure POPC while the other monolayer is a POPC/POPS mixture. Black arrows visualize the periodic boundary conditions used in this study.

and inner leaflet (104 POPC and 24 POPS) of an animal plasma membrane. The IC compartment hosts $48\left(\mathrm{~K}^{+}\right)$ counterions to neutralize the negatively charged head groups of the 48 POPS lipids, 24 per leaflet. For both models, the simulation box was adjusted around $9.0 \times 9.2 \times 22.2 \mathrm{~nm}$ after density relaxation by NPT simulations at 1 bar. In order to maximize the efficiency of the simulations, i.e., reduce the number of atoms in the system, the compartments are not equally sized. Distance between the bilayers' centers of mass (COMs) across the compartment containing the $\mathrm{AuNP}^{+}$was significantly larger in both cases, $\sim 14.9 \mathrm{~nm}$, while the other compartment was left with $\sim 7.3 \mathrm{~nm}$. The overall number of particles in both systems was around 143000 .

All simulated systems use a united atom force field which is largely compatible with the Berger force field. ${ }^{41}$ Essentially, the force field is a mixture of a tuned united OPLS (nonbonded interactions) and GROMOS (bonded interactions) force fields. The used POPC is implemented as originally developed by Berger et al. ${ }^{41,42}$ with adjustments for the double bond extracted from Bachar et al. ${ }^{43}$ The POPS force field model obeys the Mukhopadhyay et al. implementation. ${ }^{44}$ Water molecules were represented using the SPC model. ${ }^{45}$ The gold nanoparticle has been assembled as described in Heikkilä et al., ${ }^{36}$ and it is based on a realistic atomic model for $\mathrm{Au}_{144} \mathrm{SR}_{60}$ which is in agreement with experimental data. ${ }^{26} \mathrm{AuNP}^{+}$is associated with $60 \mathrm{Cl}^{-}$counterions in each compartment. In addition, $150 \mathrm{mM}$ of salt has been added to the systems in two simulations. The counterions $\mathrm{Na}^{+}, \mathrm{Cl}^{-}$, and $\mathrm{K}^{+}$use their original GROMACS-87 parameters. ${ }^{46}$

Prior to the actual production runs, all prepared systems were energy minimized. The production simulations were performed over $200 \mathrm{~ns}$ for each setup (IC and EC systems with and without salt, each). Four extra replicas of the IC and EC systems (without salt) were also simulated for $100 \mathrm{~ns}$ with different initial velocities in order to gain statistics. In addition to the freely diffusing systems, we also applied constraints to bring $\mathrm{AuNP}^{+}$in contact with the EC leaflet, and the system was simulated for $300 \mathrm{~ns}$ after releasing the constraints. (The same procedure was also carried out for IC although it is not necessary for achieving the membrane attachment.) The time step was set to $2 \mathrm{fs}$, and the neighbor list (cutoff $1.0 \mathrm{~nm}$ ) was updated for every 10th step (20 fs). Furthermore, in order to study particularly rapid processes related to water hydrogen bonds and counterion contacts between $\mathrm{AuNP}^{+}$and the surrounding solution, a set of ten $1 \mathrm{~ns} \mathrm{MD}$ simulations starting at different frames of the original simulations were performed by storing data every $0.5 \mathrm{ps}$.

The simulations were performed in the canonical NPT ensemble by setting the temperature equal to $310 \mathrm{~K}$ using the Berendsen thermostat ${ }^{47}$ with a time constant of $0.1 \mathrm{ps}$ and with a pressure coupling using the Berendsen algorithm ${ }^{47}$ with a compressibility of $4.5 \times 10^{-5} \mathrm{bar}^{-1}$, time constant of $5 \mathrm{ps}$, and reference pressure of 1 bar. Long-range electrostatic interactions were calculated using the particle-mesh Ewald summation (PME) method. ${ }^{48}$ A real space cutoff of $1.0 \mathrm{~nm}$ and a reciprocal grid of $77 \times 78 \times 189$ cells with a fourth-order $B$-spline interpolation were employed. For van der Waals interactions, a cutoff distance of $1.0 \mathrm{~nm}$ was used.

The umbrella sampling method ${ }^{49}$ was employed to calculate the free energy profile of the nanoparticle approaching the extracellular leaflet of one of the bilayers. The reaction coordinate was chosen to be the distance between the core gold atoms and the phosphorus atoms of the target leaflet in the direction normal to the bilayer $(z)$. A harmonic biasing potential was employed, and a total of 23 windows were simulated with the minimum of this biasing potential located between 0.6 and $5.0 \mathrm{~nm}$ with a spacing of $0.2 \mathrm{~nm}$. A force constant of $2000 \mathrm{~kJ} /\left(\mathrm{mol} \mathrm{nm}{ }^{2}\right)$ was chosen as this resulted in sufficient sampling of the total reaction coordinate. The windows with the nanoparticle close to the membrane interface $(0.6-3.6 \mathrm{~nm})$ were simulated for $100 \mathrm{~ns}$, whereas the rest of the windows $(3.8-5.0 \mathrm{~nm})$ were simulated for $50 \mathrm{~ns}$. The data for the last $40 \mathrm{~ns}$ were employed in the analysis for all windows. Other simulation parameters follow those of the presented equilibrium simulations. The profiles and their statistical error estimates were obtained by the GROMACS tool g_wham. ${ }^{50}$

\section{RESULTS}

AuNP $^{+}$Faces a Free Energy Barrier for Binding on the Extracellular Side but Binds Spontaneously to the Intracellular Leaflet. Four AuNP ${ }^{+}$-double membrane setups were simulated for $200 \mathrm{~ns}$ at $310 \mathrm{~K}$ : EC, EC with salt, IC, and IC with salt (Figure 2). On the IC side, $\mathrm{AuNP}^{+}$always approaches the membrane within 10-20 ns, with and without added salt (Figure 2c,d), and it attaches rapidly to the membrane in a manner where the functional amine groups 


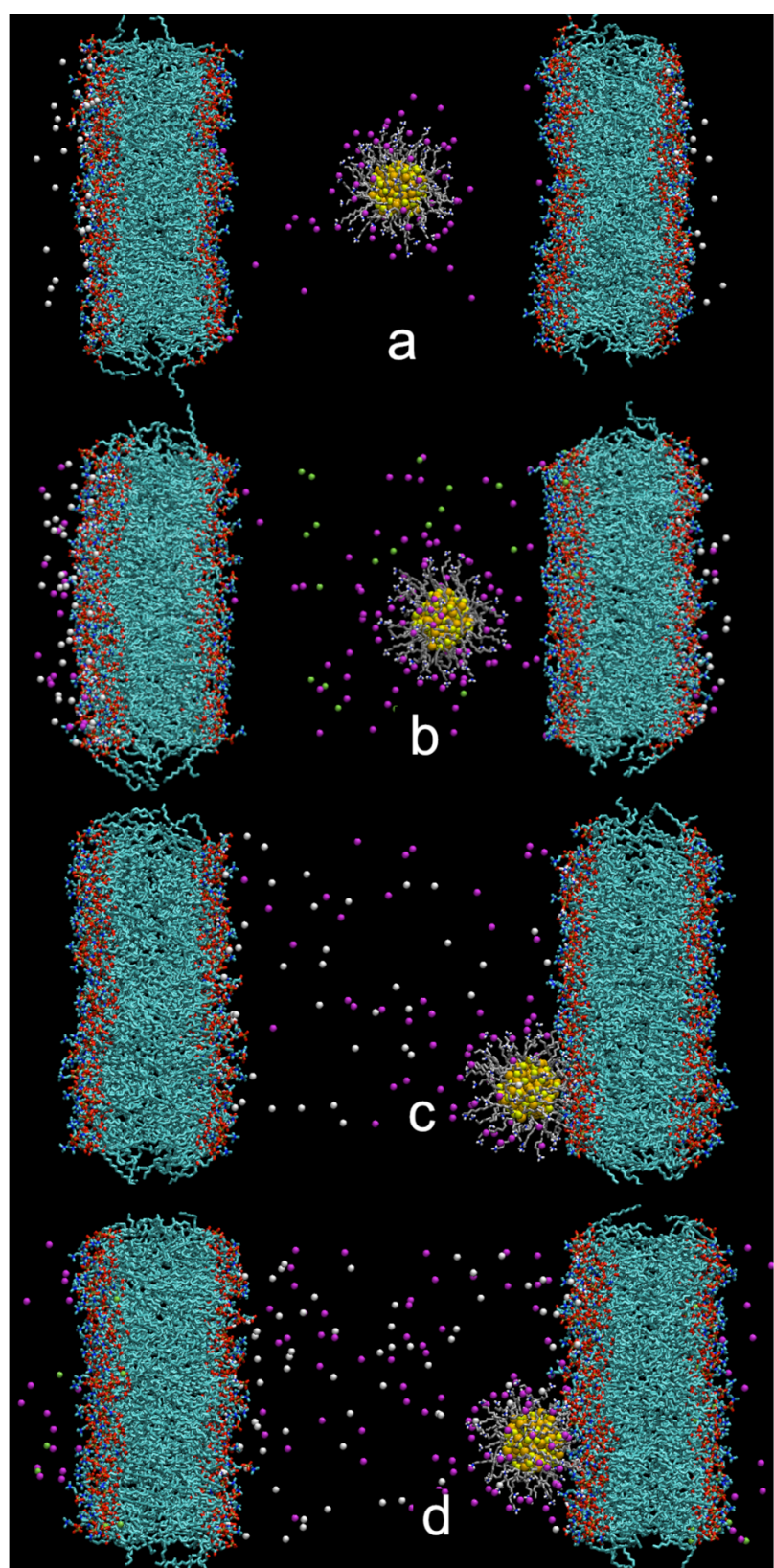

Figure 2. Visualization of $\mathrm{AuNP}^{+}$with membranes. $\mathrm{AuNP}^{+}$is placed between membranes in the extracellular (EC) and intracellular (IC) compartments. (a) EC; (b) EC with $\mathrm{NaCl}$; (c) IC; (d) IC with KCl. Color code: $\mathrm{AuNP}^{+}$[Au (orange), S (yellow), alkyl chains (gray), primary amine (blue and white)]; lipids [C (cyan), O (red), P (brown), $\mathrm{N}$ (blue)]; ions $\left[\mathrm{Na}^{+}\right.$(lime), $\mathrm{Cl}^{-}$(magenta), $\mathrm{K}^{+}$(white)].

are in contact with the membrane surface (see Figure S1 in Supporting Information). Meanwhile, $\mathrm{AuNP}^{+}$largely fluctuates between the bilayers in the EC compartment, and it does not form direct contacts with the membrane during the time scale of the simulations (Figure 2a,b and Figure S1). Four shorter simulation replicas, $100 \mathrm{~ns}$ each, for systems without extra salt and with different initial conditions were performed to validate the results, providing the same outcome as above.

The fact that $\mathrm{AuNP}^{+}$does not bind spontaneously to the EC leaflet is intriguing. We considered this binding process more carefully through free energy (umbrella sampling) simulations. The results depicted in Figure 3 highlight that there is a free energy barrier of about $11.7 \mathrm{~kJ} / \mathrm{mol}\left(4.7 k_{\mathrm{B}} T\right)$ for the binding of

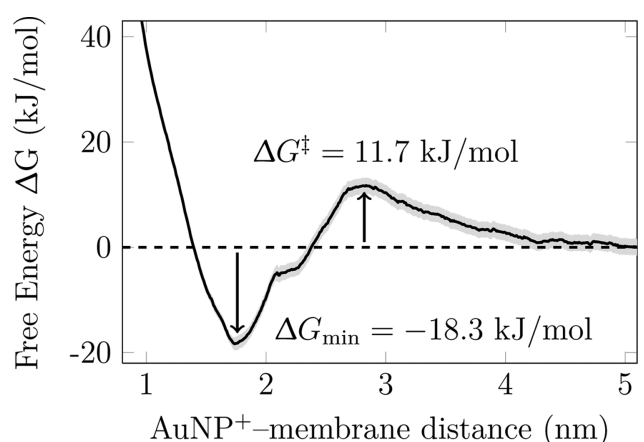

Figure 3. Free energy (umbrella sampling) profile of $\mathrm{AuNP}^{+}$ adsorption to the membrane-water interface on the EC side. The simulation conditions refer to those without additional salt. The distance is measured between the $\mathrm{AuNP}^{+}$center and membrane surface plane ( $\mathrm{P}$ atoms).

$\mathrm{AuNP}^{+}$to the EC leaflet. The free energy computations also revealed that once the barrier is crossed, the nanoparticle is stably bound to the EC leaflet at the membrane-water interface, about $1.7 \mathrm{~nm}$ from the membrane center, with a well depth of about $18.3 \mathrm{~kJ} / \mathrm{mol}\left(7.4 k_{\mathrm{B}} T\right)$. These data are in full agreement with experiments ${ }^{23}$ as discussed in detail below.

Further, the finding that $\mathrm{AuNP}^{+}$binds to the IC leaflet is also intriguing (see Figure $2 c, d$ ), as this highlights that there is a strong force driving NP to the surface of the IC leaflet. However, the nanoparticle does not spontaneously penetrate through the membrane, neither in IC nor in EC simulations. As the discussion below shows, one of the reasons causing this is the considerable charge of the AuNP ${ }^{+}$explored here. We conclude that $\mathrm{AuNP}^{+}$binds spontaneously to the IC leaflet, but on the EC side there is a free energy barrier to cross prior to NP binding.

$\mathrm{Cl}^{-}$Counterions Screen AuNP ${ }^{+}$but Do Not Inhibit Binding to the Intracellular Leaflet. The partial density profiles in the IC case show AuNP ${ }^{+}$in a stable contact with the membrane (see Figure 4a,b). Instead, the EC profiles in Figure $4 \mathrm{c}, \mathrm{d}$ show a broader distribution for $\mathrm{AuNP}^{+}$, consistent with its fluctuation around the compartment center. Each case shows a rather compact ionic cloud of $\mathrm{Cl}^{-}$around $\mathrm{AuNP}^{+}$, screening its

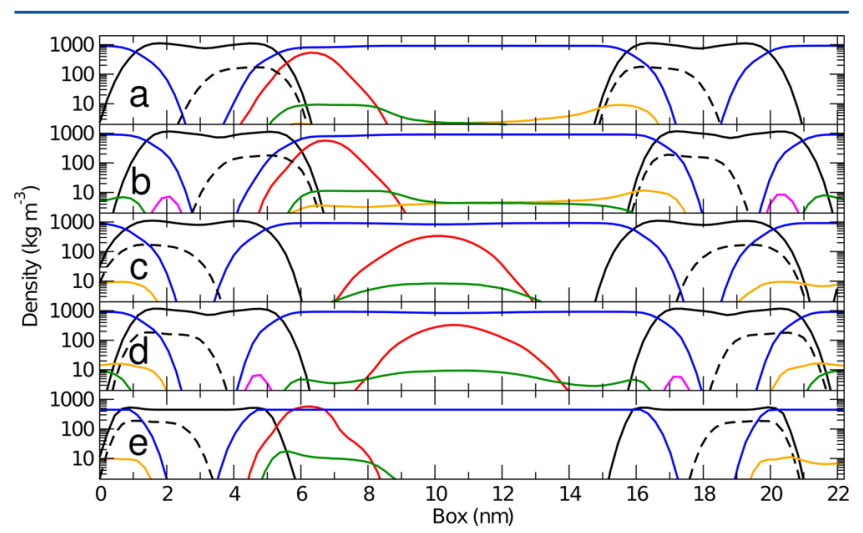

Figure 4. Partial density distributions of individual atoms/molecular groups of the AuNP ${ }^{+}$systems in (a) IC, (b) IC with salt, (c) EC, (d) EC with salt, and (e) EC constraint-released. The location $x=0$ corresponds to the side of the simulation box. Color code: membrane (black, solid), NP (red, solid), water (blue, solid), POPS (black, dashed), $\mathrm{K}^{+}$(orange, solid), $\mathrm{Cl}^{-}$(green, solid), and $\mathrm{Na}^{+}$(magenta, solid). 
positive charge. The halo pattern of $\mathrm{Cl}^{-}$in the IC compartment is not as symmetric as in EC since the anion concentration has been reduced in the membrane contact zone. Furthermore, the $\mathrm{K}^{+}$ions have accumulated close to the opposite membrane surface with respect to $\mathrm{AuNP}^{+}$in the IC compartment, and the net negative charge of the cytosolic leaflet is more exposed to the nanoparticle. The source of this $\mathrm{K}^{+}$asymmetry is the overcharging effect caused by the high surface charge density of $\mathrm{AuNP}^{+}$.

The halo pattern of counterions is highlighted in Figure 5, where the $\mathrm{Cl}^{-}$concentration has been visualized for the EC and

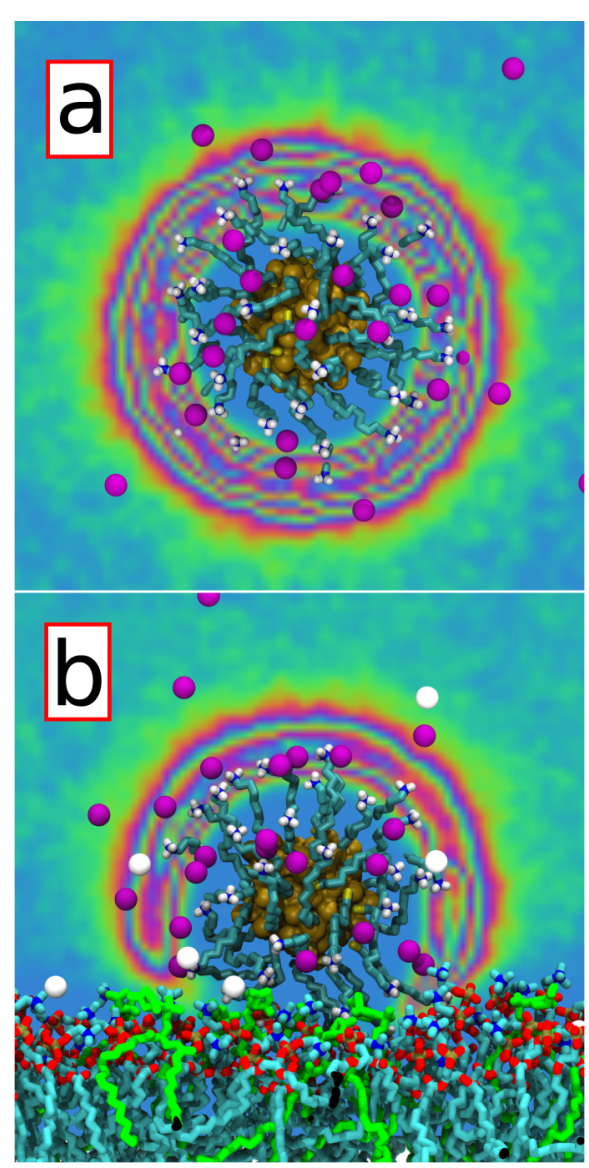

Figure 5. Visualization of $\mathrm{Cl}^{-}$counterion density around $\mathrm{AuNP}^{+}$in (a) the EC compartment (free, as in bulk-like water) and (b) the IC compartment (membrane-water interface). The counterion concentrations have been averaged over $60 \mathrm{~ns}$ simulations. Suggestive transient positions for the ions are also shown as given by the color code: $\mathrm{Cl}^{-}$(magenta) and $\mathrm{K}^{+}$(white), next to a membrane where POPS lipids (green) are shown separately. Color scale for the density from low to high: light-blue, green, yellow, orange, red, purple.

IC cases. For IC, the limited number of contacts of $\mathrm{Cl}^{-}$with the membrane, and in particular with POPS lipids, becomes evident despite the fact that there are also $\mathrm{K}^{+}$ions around. There are no $\mathrm{Cl}^{-}$counterions between the nanoparticle and the membrane as the negative charge of POPS causes repulsion. The tight space between the thiol chains of $\mathrm{AuNP}^{+}$together with a less hydrated environment makes it difficult for the relatively voluminous $\mathrm{Cl}^{-}$to penetrate into the soft surface region of $\mathrm{AuNP}^{+}$, allowing the nanoparticle surface to behave effectively as a charged wall. The terminal amino groups are distributed in equidistant positions which causes ripples in the $\mathrm{Cl}^{-}$ concentration. It is also clear that the surface charge density is not large enough to fully condense the counterions to the surface. This is expected as both $\mathrm{Cl}^{-}$and $\mathrm{NH}_{3}{ }^{+}$are monovalent and relatively small. ${ }^{51}$ Under these conditions and in agreement with the Gouy-Chapman model, one expects the formation of a diffuse anionic cloud around $\mathrm{AuNP}^{+}$, and this is indeed clearly seen in the anion density maps and more vaguely also in the $\mathrm{Cl}^{-}$density profiles along the membrane normal direction (Figure 4).

AuNP ${ }^{+}$Binds to the Extracellular Leaflet Once the Free Energy Barrier Has Been Overcome, and the Binding Is Stable and Suggestive of Pore Formation. The results discussed above do not seem to support the current experimental evidence that $\mathrm{AuNP}^{+}$originally outside a cell could spontaneously reach the cytoplasmic region ${ }^{11,12,14,19,37,38}$ or how it could self-penetrate to a cell. ${ }^{23}$ Instead, we observe in the EC compartment that the nanoparticle resists the formation of a contact with the membrane (Figure 3 ). This result is understandable considering the surface charges of the proximal leaflets. For $\mathrm{AuNP}^{+}$in IC, the surrounding membrane leaflet contains POPS which is negatively charged, and this clearly explains the observed attraction of the positively charged $\mathrm{AuNP}^{+}$. For AuNP ${ }^{+}$in $\mathrm{EC}$, the proximal leaflet is composed of pure POPC which, although being neutral as a whole, has a zwitterionic headgroup $\left(\mathrm{NH}_{3}^{+}-\left(\mathrm{CH}_{2}\right)_{2}-\mathrm{O}-\left(\mathrm{PO}_{2}\right)^{-}-\mathrm{O}-\right)$ with a positive amine group pointing toward the aqueous region. This effectively creates a positive charged layer to the membrane surface, inducing a repulsive interaction with $\mathrm{AuNP}^{+}$ (Figure 3).

Recent experimental evidence on pure synthetic DSPC membranes suggests that there is a free energy barrier for approaching AuNP ${ }^{+23}$ In these experiments, the temperature of the model system had to be risen up to $326 \mathrm{~K}$ in order to activate $\mathrm{AuNP}^{+}$to bind with the membrane. Experiments also showed that subsequent cooling down of the sample to a working temperature of $298 \mathrm{~K}$ did not terminate the binding. This suggests that once the free energy barrier has been crossed, there is a net attraction between $\mathrm{AuNP}^{+}$and the membrane. Similarly, our MD simulations highlight the presence of a free energy barrier when $\mathrm{AuNP}^{+}$approaches the EC leaflet composed of POPC lipids (see Figure 3).

Having confirmed the activated binding on the EC side, we gradually pulled $\mathrm{AuNP}^{+}$closer to the membrane using a harmonic potential. Once in contact, $0.9 \mathrm{~nm}$ from AuNP ${ }^{+}$s center of mass to the membrane surface, we released the constraint and simulated the system for $300 \mathrm{~ns}$; this system is here referred to as "EC constraint-released". We observed $\mathrm{AuNP}^{+}$to remain attached to the membrane. The corresponding partial density profiles over the last $150 \mathrm{~ns}$ are shown in Figure 4e.

Once proven that $\mathrm{AuNP}^{+}$attaches to both IC (POPC/ POPS) and EC (pure POPC) layers, the latter being an activated process, it is interesting to compare the contact area between $\mathrm{AuNP}^{+}$and each of the two layers (see Figure 6). In the IC leaflet, the average equilibrium distance between the $\mathrm{AuNP}^{+}$center of mass and the POPC/POPS leaflet (phosphorus atom in POPC) is $1.4 \pm 0.2 \mathrm{~nm}$. The contact layer shows slightly convex bending toward $\mathrm{AuNP}^{+}$, allowing the system to maximize the number of POPS contacts. AuNP then rides over the membrane surface (see Figure 6a), with occasional but clear interdigitation between the $\mathrm{AuNP}^{+}$side chains and lipid head groups. If a positive side chain of $\mathrm{AuNP}^{+}$ manages to cross the positively charged POPC choline region with the help of the negatively charged phosphatidylserine 

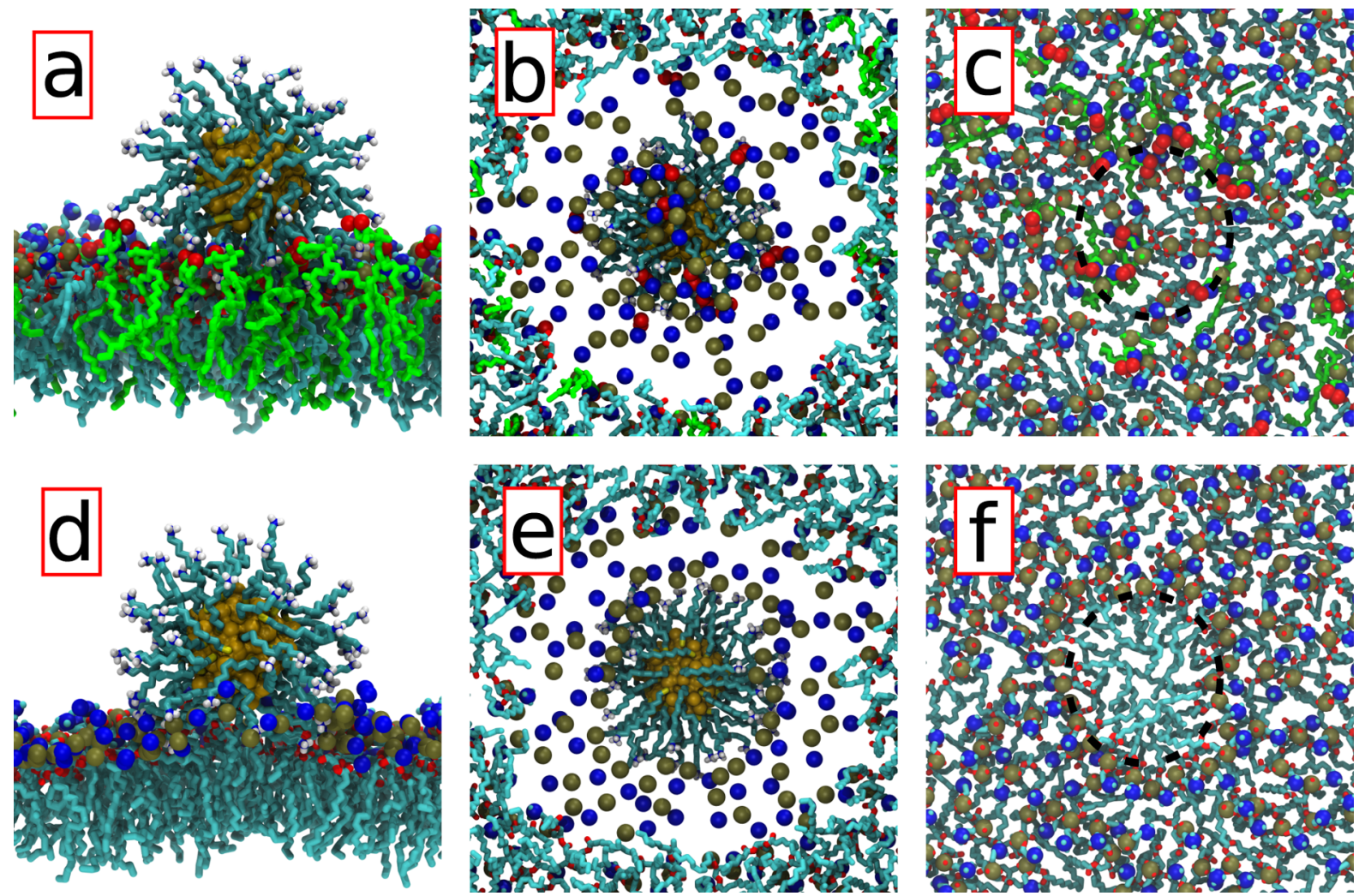

Figure 6. Morphology of the AuNP ${ }^{+}$attachment with IC (a, b, and c) and EC (d, e, and f) layers when the nanoparticle is in contact the membrane. In both cases, only the monolayer in contact with the nanoparticle is shown. Panels a and $d$ correspond to the lateral view. Panels $b$ and e depict the bottom view: here only the moieties in the head groups of POPC and POPS (not the glycerol or the hydrocarbon chains) below AuNP ${ }^{+}$are shown to monitor their effect on AuNP'. Panels $\mathrm{c}$ and $\mathrm{f}$ illustrate the top view: here AuNP ${ }^{+}$has been removed to assess its effect on the membrane (nanoparticle position shown with a black circle). AuNP ${ }^{+}$, POPS (green), and POPC are represented with rods. Head group phosphorus (khaki), amine nitrogen (blue), and serine carbonyl oxygen atoms (red) are highlighted as van der Waals spheres.

groups, it immediately becomes trapped deeper in the membrane due to the attraction of the underlying negatively charged region of the phosphate groups. Still, the most interesting feature is that $\mathrm{AuNP}^{+}$does not considerably perturb the underlying leaflet (Figure $6 \mathrm{~b}, \mathrm{c}$ ) besides slowly recruiting POPS lipids beneath itself (Figure 6a). We discuss this in more detail below.

In the EC compartment, $\mathrm{AuNP}^{+}$positions itself partly embedded in the pure POPC layer at a distance of $1.6 \pm 0.2 \mathrm{~nm}$ (phosphorus atom in POPC) (see Figure 6d). While having a larger separation distance than in IC, the nanoparticle appears to be embedded deeper in the induced concave curvature of the underlying layer. This slight membrane deformation allows the system to reduce the repulsion between the lipid head groups around $\mathrm{AuNP}^{+}$, without exposing the hydrophobic acyl chains to the water solvent. In this case no side chain interdigitation is observed, and the lipid head groups are clearly pushed away underneath $\mathrm{AuNP}^{+}$(Figure 6f). Similarly, the facing AuNP side chains bend toward the membrane plane, maximizing the number of contacts with the ring of the negatively charged phosphate groups (Figure 6e). This ring emerges because phosphatidylcholine groups strongly orient themselves, with the phosphates pointing toward $\mathrm{AuNP}^{+}$. The nanoparticle penetration into the EC leaflet results in that the corresponding electrostatic potential (EP) maps show considerable variation in the contact area for both IC and EC (see Figure S2 in Supporting Information).
Potential Mechanism for AuNP ${ }^{+}$Translocation. In contrast to the IC system, not a single $\mathrm{AuNP}^{+}$side chain points toward the hydrophobic lipid core (along membrane normal direction), and we speculate that this could be a favorable initial stage for self-penetration. The fact that $\mathrm{AuNP}^{+}$manages to isolate its charged side groups from the hydrophobic (low dielectric constant) lipid tail groups allows it to move across the membrane without a large energy penalty. The lipids in contact with $\mathrm{AuNP}^{+}$can bend toward the membrane plane as $\mathrm{AuNP}^{+}$ passes through the membrane, forming a pore. Recently reported coarse-grained simulations of $\mathrm{AuNP}^{+} \mathrm{s}$ in symmetric bilayers are in favor of this view, suggesting the formation of torus-like pores. ${ }^{33}$ In the present case, in the absence of an artificially created electrostatic potential, the driving force pulling $\mathrm{AuNP}^{+}$into the membrane is the electrostatic attraction from the negatively charged POPS lipids on the IC side, and the described mechanism should evolve until $\mathrm{AuNP}^{+}$is fully embedded in the membrane. Importantly, prior to the translocation process, it is quite obvious that $\mathrm{AuNP}^{+}$has to neutralize/deprotonate, at least in part, since there are numerous studies showing that the translocation of drugs and other charged compounds across lipid membranes has a very high free energy barrier that is lowered substantially through neutralization. ${ }^{52}$

Overall, our observations are consistent with the formation of holes ${ }^{53}$ observed in toxicity experiments. ${ }^{11,12,14,19,37,38}$ However, our observations are not fully conclusive as no $\mathrm{AuNP}^{+}$ penetration is observed during the simulations. This is due in 

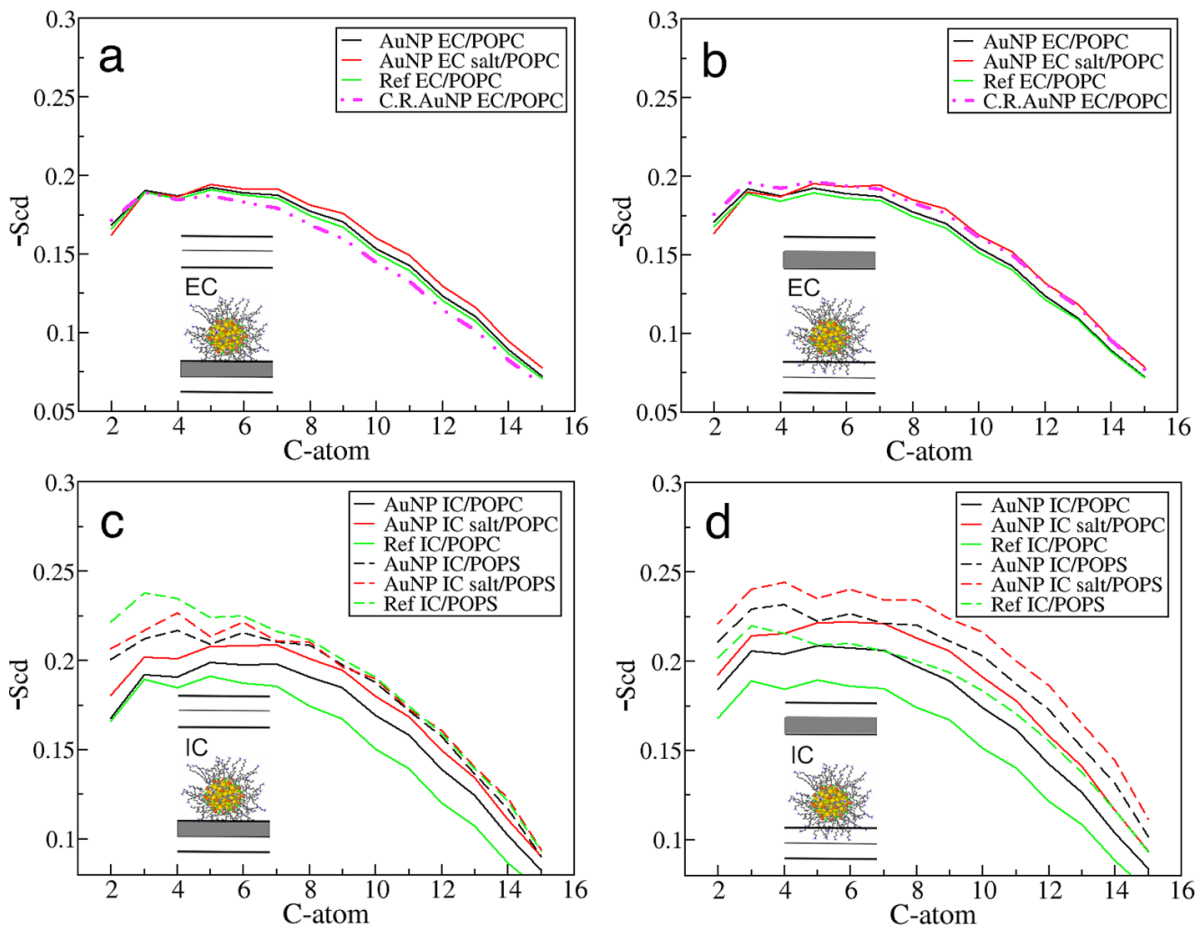

Figure 7. Order parameters $\left(-S_{\mathrm{CD}}\right)$ for the palmitoyl chains of lipids in the presence/absence of AuNP ${ }^{+}$. (a) EC leaflet close to AuNP ${ }^{+}$, data for POPC. (b) EC leaflet farther from AuNP', data for POPC. (c) IC leaflet closer to AuNP', data for POPC and POPS. (d) IC leaflet farther from $\mathrm{AuNP}^{+}$, data for POPC and POPS. Data are given for $-S_{\mathrm{CD}}$ in the AuNP ${ }^{+}$system without added salt (black), AuNP ${ }^{+}$system with added salt (red), and in the reference system without $\mathrm{AuNP}^{+}$or salt (green). Results for POPC (solid) and POPS (dashed) are shown separately. Data for the constraint-released (C.R.) simulation is depicted with a dashed magenta line. The results are based on sampling over $150 \mathrm{~ns}$ after skipping $50 \mathrm{~ns}$ for an equilibration phase. The results have been averaged over all the lipids in the leaflet in question.

part to the high charge concentration of the nanoparticle as well as the geometrical constraints inherent to the double bilayer setup used in the simulations. We discuss both of these features in the end of the article.

Structural Changes at the Extracellular and Intracellular Leaflets Are Consistent with the Proposed Mechanism. AuNP ${ }^{+}$-induced perturbations in membrane structure were determined by considering changes in the lipid hydrocarbon chain order parameter $\left(S_{\mathrm{CD}}\right)$. The results are presented in Figure 7. The order parameters for the saturated palmitoyl tail (sn-1) of both POPC and POPS were calculated for both leaflets sharing the compartment with $\mathrm{AuNP}^{+}$. The oleoyl $(s n-2)$ tail provided qualitatively the same information (data not shown). Overall, all the $S_{\mathrm{CD}}$ profiles show that on the IC side $\mathrm{AuNP}^{+}$induces increasing order for POPC, and the increase is quite substantial. In addition, increasing the salt concentration to match the biological condition $(\sim 150 \mathrm{mM})$ increases the ordering further. Meanwhile, the results also show that $\mathrm{AuNP}^{+}$affects the POPC order very little on the EC side. Only for the constraint-released case where the nanoparticle is in direct contact with the leaflet, a slight effect can be observed (Figure 7a), but the change is marginal.

For the IC compartment, overall the ordering effects of $\mathrm{AuNP}^{+}$and salt are hence significantly amplified for both POPC and POPS, but the effect depends on the region considered. In the contact layer $\mathrm{AuNP}^{+}$induces clear ordering in POPC alkyl chains, but POPS actually becomes slightly disordered close to the head groups (Figure 7c). The underlying reason for this is the interdigitation of the $\mathrm{AuNP}^{+}$ side chains in the contact layer, especially with POPS accumulated beneath the nanoparticle. In the opposite layer that is farther from the nanoparticle, we observe ordering to take place when $\mathrm{AuNP}^{+}$is present, and this effect is amplified with increasing salt (Figure $7 \mathrm{~d}$ ). The increased $\mathrm{K}^{+}$concentration close to the membrane causes this phenomenon ${ }^{54,55}$ (see Figure 4a).

The results are in agreement with the self-penetration mechanism. When AuNP ${ }^{+}$approaches the EC leaflet, the high fluidity of the lipid layer remains unaffected, and this allows quick reordering of the lipids to form a hole around AuNP ${ }^{+}{ }^{11,12,14,19,37,38}$ Instead, on the IC side the ordering increases in the contact layer, rendering this layer more impermeable. On the basis of this finding and the attractive electrostatic interaction (see Figures S1 and S2), one should expect $\mathrm{AuNP}^{+}$accumulation on the cytosolic layer.

Hydrogen Bonding Shows Binding at the Intracellular Leaflet To Be More Stable. For the overall view, it is important to discuss the stability of the nanoparticle contacts at the membrane interface. This is a topic largely related to hydrogen bonding (H-bonding) in atomic resolution and electrostatic interactions. To this end, we consider the interaction of $\mathrm{AuNP}^{+}$with water, lipids, and $\mathrm{Cl}^{-}$ions.

The average number and the lifetime of $\mathrm{H}$-bonds and ion contacts between $\mathrm{AuNP}^{+}$and solvent (Table 1) show clear differences between the IC and EC solutions: The total number of H-bonds between water and $\mathrm{AuNP}^{+}$are 141.2 \pm 3.7 and $169.7 \pm 0.1$, respectively, and also the number of ion contacts differs, $1.4 \pm 0.8$. and $4.9 \pm 0.2$. The number of water contacts is close to three per amine group in EC (60 groups), as expected, but the number of direct ion contacts is small despite the counterion cloud around the nanoparticle (see Figure 5). $\mathrm{AuNP}^{+}$attaches to the cytosolic leaflet in IC, and hence it has 
Table 1. Hydrogen Bonds and Ionic Contacts between AuNP $^{+}$and Solvent/Lipids ${ }^{a}$

\begin{tabular}{llcc} 
compartment & $\mathrm{A}$ & \multicolumn{1}{c}{$\mathrm{N}_{\mathrm{A}}$} & \multicolumn{1}{c}{$\tau_{\mathrm{A}}[\mathrm{ps}]$} \\
$\mathrm{IC}$ & $\mathrm{H}_{2} \mathrm{O}$ & $141.2 \pm 3.7$ & $10.3 \pm 0.8$ \\
& $\mathrm{Cl}^{-}$ & $1.4 \pm 0.8$ & $11.3 \pm 2.5$ \\
& lipid & $78 \pm 12$ & $99.9 \pm 19.7$ \\
$\mathrm{IC}(0.15 \mathrm{M} \mathrm{KCl})$ & $\mathrm{H}_{2} \mathrm{O}$ & $137.3 \pm 4.1$ & $9.5 \pm 1.3$ \\
& $\mathrm{Cl}^{-}$ & $3.6 \pm 0.8$ & $6.4 \pm 1.4$ \\
& lipid & $96 \pm 12$ & $102.6 \pm 15.0$ \\
EC & $\mathrm{H}_{2} \mathrm{O}$ & $169.7 \pm 0.1$ & $5.8 \pm 0.2$ \\
& $\mathrm{Cl}^{-}$ & $4.9 \pm 0.2$ & $5.5 \pm 0.3$ \\
& lipid & & \\
EC $(0.15 \mathrm{M} \mathrm{NaCl})$ & $\mathrm{H}_{2} \mathrm{O}$ & $169.7 \pm 0.1$ & $5.8 \pm 0.1$ \\
& $\mathrm{Cl}^{-}$ & $3.4 \pm 0.2$ & $5.0 \pm 0.6$ \\
& lipid & & \\
EC (C.R.) & $\mathrm{H}_{2} \mathrm{O}$ & $138.6 \pm 1.7$ & $11.9 \pm 0.7$ \\
& $\mathrm{Cl}^{-}$ & $7.3 \pm 0.3$ & $8.0 \pm 1.0$ \\
& lipid & $18 \pm 6$ & $224.3 \pm 42.7$
\end{tabular}

${ }^{a} N_{\mathrm{A}}$ is the average number of hydrogen bonds and contacts, and $\tau_{\mathrm{A}}$ is the average lifetime of the contacts, per solvent molecule/lipid. EC and IC are extracellular and intracellular compartments, respectively. C.R. refers to the EC constraint-released case.

fewer available terminal groups to make contacts with water and/or counterions. This is reflected not only in the number of $\mathrm{H}$-bonds but also in contact lifetimes, which are considerably larger in IC. AuNP ${ }^{+}$moves freely in EC, whereas its movement is restricted in IC by the interactions with the membrane-the contacts are less interrupted and consequently last longer. For $\mathrm{EC}$, the values of the number of contacts and lifetimes are similar to those in Heikkilä et al. ${ }^{36}$ for the same $\mathrm{AuNP}^{+}$in aqueous solution without the presence of bilayers, $170.8 \pm 0.2$ and $6.5 \pm 0.2 \mathrm{ps}$, respectively. Adding $150 \mathrm{mM}$ of salt $(\mathrm{KCl}$, $\mathrm{NaCl}$ ) to the systems does not significantly affect the $\mathrm{H}$-bonds between $\mathrm{AuNP}^{+}$and solvent. The AuNP-membrane contacts are numerous due to the $\mathrm{NH}_{3}{ }^{+}$groups, and their lifetimes are of the order 100 ps, illustrating that AuNP moves relatively slowly along the membrane surface.

The situation changes in $\mathrm{EC}$ when $\mathrm{AuNP}^{+}$is pulled onto the POPC leaflet (constraint-released case). The interaction with water has decreased as the effective solvent interface of $\mathrm{AuNP}^{+}$ is smaller, whereas the number of $\mathrm{Cl}^{-}$contacts is larger, reflecting changes in the dielectric medium (water/membrane) and $\mathrm{AuNP}^{+}$screening charge. Comparing with the IC case, $\mathrm{Cl}^{-}$ contact values are significantly higher, corresponding to a tighter $\mathrm{Cl}^{-}$cloud around $\mathrm{AuNP}^{+}$in the $\mathrm{EC}$ constraint-released system. Despite being attached to the membrane, there are not many H-bonds forming between $\mathrm{AuNP}^{+}$and the lipid head groups in accordance with Figure $6 \mathrm{~d}-\mathrm{f}$.

Summarizing, the analysis based on H-bonding and contacts indicates that when $\mathrm{AuNP}^{+}$is bound to a membrane, it is more stable at the IC side compared to the EC leaflet.

Why We Have Not Observed Spontaneous Permeation in the Simulations? It is clear that this is, in part, due to the high charge of $\mathrm{AuNP}^{+}$studied here. Regardless of the environment, $\mathrm{AuNP}^{+}$is always equally charged $(+60 \mathrm{e})$. A more realistic model to consider penetration would include deprotonation of the amine groups while $\mathrm{AuNP}^{+}$passes through the low dielectric lipid tail region (see discussion below). Based on earlier simulations, it is clear that deprotonation (neutralization of the nanoparticle) would strongly promote permeation across the membrane. ${ }^{52}$ However, consideration of this topic is out of the scope of this work.

The substantial charge of the nanoparticle is a seemingly simple reason to explain why no translocation was observed in simulations. This view is supported by the fact that in experiments the coverage of positively charged alkyl chains rarely exceed $70 \%$ in contrast to $100 \%$ in our case. Also, the terminal choline groups are significantly more bulky than our small amine groups, and the positive charge is more delocalized. However, according to experimental results, also cationic Au nanoparticles with terminal groups which cannot deprotonate have proven to be capable of trespassing the membrane. ${ }^{23}$ Therefore, deprotonation of the amine groups can explain the experimentally observed translocation rate only partially.

As for methodological matters related to simulations only, the use of the double bilayer setup hampers the penetration process as the lateral dimensions of the two membranes are coupled to each other via the simulation box and the periodic boundary conditions used (lateral strain). These imply that in the simulations we have done the bilayer through which the nanoparticle is translocating is not in a tensionless state, as it should be, and the tension arising from the use of the double bilayer setup will certainly increase the translocation free energy barrier. This issue could be minimized by using membrane systems much larger than the one employed in this work, but we consider it to be beyond the scope of this work. It should be noted that recent CG simulations using a double bilayer model (1032 lipids per membrane) achieved $\mathrm{AuNP}^{+}$translocation by using an external electric field of $-1.5 \mathrm{eV}$, mimicking the transmembrane potential, ${ }^{33}$ in favor of the views discussed above.

\section{CONCLUSIONS}

The nanoparticle-membrane interaction is very relevant for studying biomedical applications and potential toxic effects of nanomaterials. In this work, we have performed atomistic MD simulations of cationic gold nanoparticles $\left(\mathrm{AuNP}^{+}\right)$in both extracellular (EC) and intracellular (IC) environments in order to test their tendency to penetrate through the cell membrane and shed light on the molecular mechanisms involved. For this purpose, we have applied a double asymmetric bilayer system, using animal plasma-like membranes, with two solvent compartments (EC and IC). Our theoretical approach is unique, and the double bilayer setup differs from most previous coarse-grained simulations. The atomistic description enables us to unlock the roles of the underlying interactions and chemical features in considerable detail.

All simulations show unequivocally that electrostatics drives $\mathrm{AuNP}^{+}$to move fast toward the negatively charged surface of the cytosolic leaflet, while it experiences a free energy barrier to bind with the extracellular leaflet, in agreement with experiments. ${ }^{23}$ These results are understandable considering the opposite overall surface charge in the two leaflets. While the surface charge in the IC leaflet is dominated by the negative charge of the POPS headgroup ( $\sim 20 \%$ of the lipids in the leaflet), in the case of the EC leaflet constituted by pure zwitterionic POPC, there is a local positive surface charge due to the positively charged choline groups facing water solvent. In other words, the distinct behavior, selectivity, arises from the specific lipid composition of each leaflet.

The results also highlight the active role of ions regulating the binding with a membrane. The counterions not only play a critical role by shielding the large charge of $\mathrm{AuNP}^{+}$during the 
transit to the membrane, but they also foster the process by migration to compensate for electrostatic potential imbalances. The role of counterions in this context was most evident on the IC side, and this process was further supported by POPS lipids that were observed to play an active role by slightly popping out of the membrane and capturing the nanoparticle, as the membrane reorganized through lateral diffusion of POPS to concentrate right underneath $\mathrm{AuNP}^{+}$.

As mentioned above, $\mathrm{AuNP}^{+}$was found to experience a free energy barrier to bind with the EC leaflet, in agreement with experiments. $^{23}$ This may appear puzzling given that several in vivo experiments have shown $\mathrm{AuNP}^{+}$to interact with the $\mathrm{EC}$ layer spontaneously. ${ }^{11,12,14,19,37,38}$ However, in real plasma membranes other membrane bound components, such as the negatively charged glycocalyx, ${ }^{56}$ can play a similar role as POPS in the cytosolic compartment, thus facilitating the contact of $\mathrm{AuNP}^{+}$and the EC layer.

What would be a plausible mechanism for $\mathrm{AuNP}^{+}$ permeation? AuNP ${ }^{+}$attaches to both the IC (POPC/POPS) and EC (POPC) layers in a stable manner, the latter taking place after the crossing of a free energy barrier. However, the nature of $\mathrm{AuNP}^{+}$membrane interaction differs significantly.

On the EC side, there are no lipid head groups beneath the nanoparticle as they migrate to the boundary of the $\mathrm{AuNP}^{+}$ contact region and orient themselves with the phosphate groups pointing toward $\mathrm{AuNP}^{+}$. The side chains of $\mathrm{AuNP}^{+}$also bend toward the membrane plane to be in contact with the surface phosphates. As a result, the $\mathrm{AuNP}^{+}$interacts with the EC membrane in a very singular way: Below the nanoparticle, hydrophobic interactions of the lipid tails and the mainly hydrophobic alkanethiol tails of $\mathrm{AuNP}^{+}$prevail, while in the borders of its projection there are ionic contacts between $\mathrm{AuNP}^{+}$amine terminal groups and oriented phosphate groups of POPC. This membrane reorganization gives room for the formation of a patch that is largely hydrophobic. Meanwhile, on the IC side, there is interdigitation between $\mathrm{AuNP}^{+}$side chains and lipid head groups and considerable enrichment of phosphatidylserines under AuNP ${ }^{+}$.

We speculate that together these constitute the initial stage for pore formation, possibly having the torus shape ${ }^{33,53}$ seen in experiments. ${ }^{11,12,14,19,37,38}$ After AuNP ${ }^{+}$has adsorbed to the EC leaflet, at least partial deprotonation of the amine groups will occur before permeation takes place through formation of a pore. The proposed mechanism could be tested in experiments by decreasing the length of $\mathrm{AuNP}^{+}$side chains and monitoring the hole formation/toxicity. Presumably, shorter side chains will result in less holes/lower toxicity as the initial stages of the pore formation is hampered.

Combining these results, we propose that $\mathrm{AuNP}^{+}$is able to approach and attach to the plasma membrane aided by negatively charged membrane-bound components, e.g. glycocalyx. After this, $\mathrm{AuNP}^{+}$can form a large pore while moving toward the cytosolic layer, and we expect that the cytotoxicity of $\mathrm{AuNP}^{+}$arises here. The nanoparticle can simply destabilize the membrane as seen in experiments ${ }^{23}$ or favor the diffusion of phosphatidylserine lipids from the cytoplasmic leaflet to the extracellular one through the pore boundaries, which can potentially initiate apoptosis. Finally, in case $\mathrm{AuNP}^{+}$manages to permeate all the way to the cytoplasm, it will strongly attach to the inner IC leaflet and accumulate. At this point, increasing nanoparticle concentration is expected to disturb many critical molecular functions due to the high $\mathrm{AuNP}^{+}$charge which can alter membrane protein conformations.

\section{ASSOCIATED CONTENT}

\section{Supporting Information}

Additional data for computational methods, nanoparticle distance from the membrane as a function of time for several MD simulations, and electrostatic potential profiles as contour plots of the $3 \mathrm{D}$ distributions. This material is available free of charge via the Internet at http://pubs.acs.org.

\section{AUTHOR INFORMATION}

\section{Corresponding Author}

*E-mail: jaakko.akola@tut.fi (J.A.).

\section{Author Contributions}

$\nabla_{\text {E.H. }}$ and H.M.-S. contributed equally to the article.

Notes

The authors declare no competing financial interest.

\section{ACKNOWLEDGMENTS}

We thank Pu-Chun Ke for inspiring discussions. The computations were performed on the Juropa (Intel Xeon 5570) and Cray XT4/XT5 computers in the Forschungszentrum Jülich (Germany) and CSC - IT Centre for Science Ltd (Espoo, Finland). Financial support is provided from the Academy of Finland through its Centre of Excellence Programs (Project 251748, E. Heikkilä and J. Akola; Project 272130, H. Martinez-Seara, M. Javanainen and I. Vattulainen). H. Häkkinen is funded through the Academy of Finland project 128341, and I. Vattulainen acknowledges support from the European Research Council (Advanced Grant CROWDEDPRO-LIPIDS).

\section{REFERENCES}

(1) Leifert, A.; Pan-Bartnek, Y.; Simon, U.; Jahnen-Dechent, W. Molecularly Stabilised Ultrasmall Gold Nanoparticles: Synthesis, Characterization and Bioactivity. Nanoscale 2013, 5, 6224-6242.

(2) Shichibu, Y.; Negishi, Y.; Tsunoyama, H.; Kanehara, M.; Teranishi, T.; Tsukuda, T. Extremely High Stability of Glutathionate-Protected $\mathrm{Au}_{25}$ Clusters Against Core Etching. Small 2007, 3, 835-839.

(3) Lopez-Acevedo, O.; Kacprzak, K. A.; Akola, J.; Häkkinen, H. Quantum Size Effects in Ambient CO Oxidation Catalysed by LigandProtected Gold Clusters. Nat. Chem. 2010, 2, 329-334.

(4) Murphy, C. J.; Anand, M. G.; Stone, J. W.; Sisco, P. N.; Alkinaly, A. M.; Goldsmith, E. C.; Baxter, S. C. Gold Nanoparticles in Biology: Beyond Toxicity to Cellular Imaging. Acc. Chem. Res. 2008, 41, 17211730.

(5) Daniel, M.; Astruc, D. Gold Nanoparticles: Assembly, Supramolecular Chemistry, Quantum-Size-Related Properties, and Applications Toward Biology, Catalysis, and Nanotechnology. Chem. Rev. 2004, 104, 293-346.

(6) Sperling, R. A.; Gil, P. R.; Zhang, F.; Zanella, M.; Parak, W. J. Biological Applications of Gold Nanoparticles. Chem. Soc. Rev. 2008, 37, 1896-1908.

(7) Jin, R. Quantum Sized Thiolate-Protected Gold Nanoclusters. Nanoscale 2010, 2, 343-362.

(8) Dykman, L.; Khlebtsov, N. Gold Nanoparticles in Biomedical Applications: Recent Advances and Perspectives. Chem. Soc. Rev. 2012, 41, 2256-2282.

(9) Dreaden, E. C.; Alkilany, A. M.; Huang, X.; Murphy, C. J.; ElSayed, M. A. The Golden Age: Gold Nanoparticles for Biomedicine. Chem. Soc. Rev. 2012, 41, 2740-2779.

(10) Nel, A.; Xia, T.; Mädler, L.; Ning, L. Toxic Potential of Materials at the Nanolevel. Science 2006, 622-627.

(11) Khlebtsov, N.; Dykman, L. Biodistribution and Toxicity of Engineered Gold Nanoparticles: A Review of in Vitro and in Vivo Studies. Chem. Soc. Rev. 2011, 40, 1647-1671. 
(12) Hirano, A.; Yoshikawa, H.; Matsushita, S.; Yamada, Y.; Shiraki, K. Adsorption and Disruption of Lipid Bilayers by Nanoscale Protein Aggregates. Langmuir 2012, 28, 3887-3895.

(13) Álvarez, Y. D.; Fauerbach, J. A.; Pellegrotti, J. V.; Jovin, T. M.; Jares-Erijman, E. A.; Stefani, F. D. Influence of Gold Nanoparticles on the Kinetics of a-Synuclein Aggregation. Nano Lett. 2013, 13, 61566163.

(14) Goodman, C. M.; McCusker, C. D.; Yilmaz, T.; Rotello, V. M. Toxicity of Gold Nanoparticles Functionalized with Cationic and Anionic Side Chains. Bioconjugate Chem. 2004, 15, 897-900.

(15) Lewinski, N.; Colvin, V.; Drezek, R. Cytotoxicity of Nanoparticles. Small 2008, 4, 26-49.

(16) Alkilany, A. M.; Nagaria, P. K.; Hexel, C. R.; Shaw, T. J.; Murphy, C. J.; Wyatt, M. D. Cellular Uptake and Cytotoxicity of Gold Nanorods: Molecular Origin of Cytotoxicity and Surface Effects. Small 2009, 5, 701-708.

(17) Pan, Y.; Leifert, A.; Ruau, D.; Neuss, S.; Bornemann, J.; Schmid, G.; Brandau, W.; Simon, U.; Jahnen-Dechent, W. Gold Nanoparticles of Diameter $1.4 \mathrm{~nm}$ Trigger Necrosis by Oxidative Stress and Mitochondrial Damage. Small 2009, 5, 2067-2076.

(18) Verma, A.; Uzun, O.; Hu, Y.; Hu, Y.; Han, H.-S.; Watson, N.; Chen, S.; D.J, I.; Stellacci, F. Surface-Structure-Regulated CellMembrane Penetration by Monolayer-Protected Nanoparticles. Nat. Mater. 2008, 7, 588-595.

(19) Mecke, A.; Lee, D.-K.; Ramamoorthy, A.; Orr, B. G.; Banaszak Holl, M. M. Synthetic and Natural Polycationic Polymer Nanoparticles Interact Selectively with Fluid-Phase Domains of DMPC Lipid Bilayers. Langmuir 2005, 21, 8588-8590.

(20) Leroueil, P. R.; Berry, S. A.; Duthie, K.; Han, G.; Rotello, V. M.; McNerny, D. Q.; Baker, J. R., Jr.; Orr, B. G.; Banaszak Holl, M. M. Wide Varieties of Cationic Nanoparticles Induce Defects in Supported Lipid Bilayers. Nano Lett. 2008, 8, 420-424.

(21) Chen, J.; Hessler, J. A.; Putchakayala, K.; Panama, B. K.; Khan, D. P.; Hong, S.; Mullen, D. G.; DiMaggio, S. C.; Som, A.; Tew, G. N.; Lopatin, A. N.; Baker, J. R.; Banaszak Holl, M. M.; Orr, B. G. Cationic Nanoparticles Induce Nanoscale Disruption in Living Cell Plasma Membranes. J. Phys. Chem. B 2009, 113, 11179-11185.

(22) Van Lehn, R.; Atukorale, P. U.; Carney, R. P.; Yang, Y.-S.; Stellacci, F.; Irvine, D. J.; Alexander-Katz, A. Effect of Particle Diameter and Surface Composition on the Spontaneous Fusion of Monolayer-Protected Gold Nanoparticles with Lipid Bilayers. Nano Lett. 2013, 13, 4060-4067.

(23) Tatur, S.; Maccarini, M.; Barker, R.; Nelson, A.; Fragneto, G. Effect of Functionalized Gold Nanoparticles on Floating Lipid Bilayers. Langmuir 2013, 29, 6606-6614.

(24) Fields-Zinna, C. A.; Sardar, R.; Beasley, C. A.; Murray, R. W. Electrospray Ionization Mass Spectrometry of Intrinsically Cationized

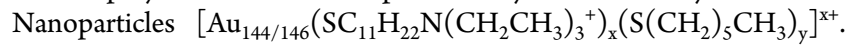
J. Am. Chem. Soc. 2009, 131, 16266-16271.

(25) Qian, H.; Jin, R. Controlling Nanoparticles with Atomic Precision: The Case of $\mathrm{Au}_{144}\left(\mathrm{SCH}_{2} \mathrm{CH}_{2} \mathrm{Ph}\right)_{60}$. Nano Lett. 2009, 9, 4083-4087.

(26) Lopez-Acevedo, O.; Akola, J.; Whetten, R.; Grönbeck, H.; Häkkinen, H. Structure and Bonding in the Ubiquitous Icosahedral Metallic Gold Cluster $\mathrm{Au}_{144}(\mathrm{SR})_{60}$. J. Phys. Chem. C 2009, 113, 50355038.

(27) Qian, H.; Jin, R. Ambient Synthesis of $\mathrm{Au}_{144}(\mathrm{SR})_{60}$ Nanoclusters in Methanol. Chem. Mater. 2011, 23, 2209-2217.

(28) Li, Y.; Chen, X.; Gu, N. Computational Investigation of Interaction between Nanoparticles and Membranes: Hydrophobic/ Hydrophilic Effect. J. Phys. Chem. B 2008, 112, 16647-16653.

(29) Li, Y.; Gu, N. Thermodynamics of Charged Nanoparticle Adsorption on Charge-Neutral Membranes: A Simulation Study. J. Phys. Chem. B 2010, 114, 2749-2754.

(30) Lin, J.; Zhang, H.; Chen, Z.; Zheng, Y. Penetration of Lipid Membranes by Gold Nanoparticles: Insights into Cellular Uptake, Cytotoxicity, and Their Relationship. ACS Nano 2010, 4, 5421-5429.
(31) Li, Y.; Li, X.; Li, Z.; Gao, H. Surface-Structure-Regulated Penetration of Nanoparticles across a Cell Membrane. Nanoscale 2012, 4, 3768-3775.

(32) Van Lehn, R. C.; Alexander-Katz, A. Free Energy Change for Insertion of Charged, Monolayer-Protected Nanoparticles into Lipid Bilayers. Soft Matter 2014, 10, 648-658.

(33) Lin, J.; Alexander-Katz, A. Cell Membranes Open "Doors" for Cationic Nanoparticles/Biomolecules: Insights into Uptake Kinetics. ACS Nano 2013, 7, 10799-10808.

(34) Li, Y.; Yang, Z.; Hu, N.; Zhou, R.; Chen, X. Insights into Hydrogen Bond Dynamics at the Interface of the Charged MonolayerProtected Au Nanoparticle from Molecular Dynamics Simulation. J. Chem. Phys. 2013, 138, 184703-1-9.

(35) Van Lehn, R. C.; Alexander-Katz, A. Structure of MixedMonolayer-Protected Nanoparticles in Aqueous Salt Solution from Atomistic Molecular Dynamics Simulations. J. Phys. Chem. C 2013, 117, 20104-20115.

(36) Heikkilä, E.; Gurtovenko, A.; Martinez-Seara, H.; Vattulainen, I.; Häkkinen, H.; Akola, J. Atomistic Simulations of Functional $\mathrm{Au}_{144}(\mathrm{SR})_{60}$ Gold Nanoparticles in Aqueous Environment. J. Phys. Chem. C 2012, 116, 9805-9815.

(37) Ruenraroengsak, P.; Novak, P.; Berhanu, D.; Thorley, A. J.; Valsami-Jones, E.; Gorelik, J.; Korchev, Y. E.; Tetley, T. D. Respiratory Epithelial Cytotoxicity and Membrane Damage (Holes) Caused by Amine-Modified Nanoparticles. Nanotoxicology 2012, 6, 94-108.

(38) Banaszak Holl, M. M. Nanotoxicology: A Personal Perspective. WIREs Nanomed. Nanobiotechnol. 2009, 1, 353-359.

(39) Gurtovenko, A. A. Asymmetry of Lipid Bilayers Induced by Monovalent Salt: Atomistic Molecular Dynamics Study. J. Chem. Phys. 2005, 122, 244902.

(40) Van Der Spoel, D.; Lindahl, E.; Hess, B.; Groenhof, G.; Mark, A. E.; Berendsen, H. J. C. GROMACS: Fast, Flexible and Free. J. Comput. Chem. 2005, 26, 1701-1718.

(41) Berger, O.; Edholm, O.; Jähnig, F. Molecular Dynamics Simulations of a Fluid Bilayer of Dipalmitoylphosphatidylcholine at Full Hydration, Constant Pressure, and Constant Temperature. Biophys. J. 1997, 72, 2002-2013.

(42) Tieleman, D. P.; Berendsen, H. J. C. A Molecular Dynamics Study of the Pores Formed by Escherichia Coli OmpF Porin in a Fully Hydrated Palmitoyloleoylphosphatidylcholine Bilayer. Biophys. J. 1998, $74,2786-2801$.

(43) Bachar, M.; Brunelle, P.; Tieleman, D. P.; Rauk, A. Molecular Dynamics Simulation of a Polyunsaturated Lipid Bilayer Susceptible to Lipid Peroxidation. J. Phys. Chem. B 2004, 108, 7170-7179.

(44) Mukhopadhyay, P.; Monticelli, L.; Tieleman, D. P. Molecular Dynamics Simulation of a Palmitoyl-Oleoyl Phosphatidylserine Bilayer with $\mathrm{Na}^{+}$Counterions and $\mathrm{NaCl}$. Biophys. J. 2004, 86, 1601-1609.

(45) Berendsen, H. J. C.; Postma, J. P. M.; van Gunsteren, W. F.; Hermans, J. In Intermolecular Forces; Pullman, B., Ed.; Reidel: Dordrecht, 1981; Chapter Interaction models for water in relation to protein hydration.

(46) Tieleman, D. P.; Berendsen, H. J. C. Molecular Dynamics Simulations of a Fully Hydrated Dipalmitoylphosphatidylcholine Bilayer with Different Macroscopic Boundary Conditions and Parameters. J. Chem. Phys. 1996, 105, 4871-4880.

(47) Berendsen, H. J. C.; Postma, J. P. M.; Van Gunsteren, W. F.; Dinola, A.; Haak, J. R. Molecular Dynamics with Coupling to an External Bath. J. Chem. Phys. 1984, 81, 3684-3690.

(48) Essmann, U. L.; Perera, L.; Berkowitz, M. L.; Darden, T.; Lee, H.; Pedersen, L. G. A. Smooth Particle Mesh Ewald Method. J. Chem. Phys. 1995, 103, 8577-8593.

(49) Torrie, G. M.; Valleau, J. P. Nonphysical Sampling Distributions in Monte Carlo Free-Energy Estimation: Umbrella Sampling. J. Comput. Phys. 1977, 23, 187-199.

(50) Hub, J. S.; de Groot, B. L.; van der Spoel, D. g_wham - A Free Weighted Histogram Analysis Implementation Including Robust Error and Autocorrelation Estimates. J. Chem. Theory Comput. 2010, 6, $3713-3720$. 
(51) Lund, M.; Jagoda-Cwiklik, B.; Woodward, C. E.; Vácha, R.; Jungwirth, P. Dielectric Interpretation of Specificity of Ion Pairing in Water. J. Phys. Chem. Lett. 2010, 1, 300-303.

(52) Cramariuc, O.; Rog, T.; Javanainen, M.; Monticelli, L.; Polishchuk, A. V.; Vattulainen, I. Mechanism for Translocation of Fluoroquinolones across Lipid Membranes. Biochim. Biophys. Acta 2012, 1818, 2563-2571.

(53) Gurtovenko, A. A.; Anwar, J.; Vattulainen, I. Defect-Mediated Trafficking across Cell Membranes: Insights from In Silico Modelling. Chem. Rev. 2010, 110, 6077-6103.

(54) Gurtovenko, A. A.; Vattulainen, I. Effect of $\mathrm{NaCl}$ and $\mathrm{KCl}$ on Phosphatidylcholine and Phosphatidylethanolamine Lipid Membranes: Insight from Atomic-Scale Simulations for Understanding Salt-Induced Effects in the Plasma Membrane. J. Phys. Chem. B 2008, 112, 1953-1962.

(55) Magarkar, A.; Karakas, E.; Stepniewski, M.; Rog, T.; Bunker, A. Molecular Dynamics Simulation of PEGylated Bilayer Interacting with Salt Ions: A Model of the Liposome Surface in the Bloodstream. J. Phys. Chem. B 2012, 116, 4212-4219.

(56) Tarbell, J. M.; Pahakis, M. Y. Mechanotransduction and the Glycocalyx. J. Int. Med. 2006, 259, 339-350. 